\title{
Neuronavigation-guided Endoscopic Endonasal Excision of Schwannoma-like Chordoma of Meckel's Cave: A Case Report
}

\author{
Ali AKAY'1, Sercan GODE², M. Sedat CAGLI \\ ${ }^{1}$ Kent Hospital, Department of Neurosurgery, Izmir, Turkey \\ ${ }^{2}$ Ege University, School of Medicine, Department of Otolaryngology, Izmir, Turkey \\ ${ }^{3}$ Ege University, School of Medicine, Department of Neurosurgery, Izmir, Turkey \\ This manuscript was presented as an e-poster presentation at the Turkish Neurosurgical Society's $31^{\text {th }}$ Annual Scientific Congress, Antalya, Turkey and \\ was published in abstract form in the proceedings of the congress, in 2017.
}

Corresponding author: Ali AKAY dr.aliakay@gmail.com

\section{ABSTRACT}

Chordomas are locally aggressive malignant tumors due to their recurrence potential and originate from embryonic notochord remnants. Chordomas can originate anywhere on the axial skeleton. They are extradural and spread by bone destruction. Chordomas are locally aggressive tumors that invade the dura mater, and may also present with secondary intradural growth. The Meckel's cave location of chordomas has been very rarely reported in the literature. Chordomas located in Meckel's cave can be radiologically confused with trigeminal schwannomas. Herein, we report a case of Meckel's cave chordoma that was successfully excised through neuronavigation-guided endoscopic endonasal excision, a technique commonly used in skull base surgeries.

KEYWORDS: Chordoma, Endoscopic endonasal, Meckel's cave, Skull base

\section{INTRODUCTION}

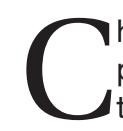
hordomas are midline tumors that develop from primitive notochord remnants (2). The incidence of

these tumors is $1 \%$ in intracranial tumors and $4 \%$ in tumors of the bone $(5,8)$. The most frequent localizations are the sacrococcygeal region (30 to 50\%), spheno-occipital region (30 to $35 \%$ ) and vertebral region (15 to $30 \%$ ). Chordomas usually develop in the cranium from the sphenooccipital synchondrosis of the clivus, but may also develop from the lower clivus and dorsum sellae. They are extradural and are spread by bone destruction. Chordomas are locally aggressive tumors that invade the dura mater, and they may also present with secondary intradural growth $(10,14)$. However, chordomas have been also shown in the literature to present with primary intradural development, although rarely $(4,15)$. Intradural chordomas most commonly demonstrate prepontine localization $(1,6,13,15)$. The prognosis of intradural chordomas is better than that of extradural chordomas. Recurrence is rare due to the absence of bone invasive and the possibility of total (en-b/oc) removal $(1,3,6,13)$. One of the rarely reported localizations for intradural or extradural chordomas is Meckel's cave (MC). Herein, we present a rare case of extradural chordoma of Meckel's cave.

\section{CASE REPORT}

A 41-year-old female patient presented with double vision for the past four months. Cranial magnetic resonance imaging (MRI) showed a massive lesion destroying the petrous bone and the left lateral wall of the clivus with heterogeneous contrast in the left Meckel's cave (Figure 1A-C). Neurological examination demonstrated an outward visual limitation in the left eye, and sixth cranial nerve palsy. The preliminary radiological diagnosis of the mass lesion was schwannoma. The patient underwent neuronavigation-guided endoscopic endonasal tumor excision. In the postoperative cranial MRI, the mass lesion was observed to be totally excised (Figure $2 A-D)$. During the intraoperative observation, it was suggested that the pathology of the mass lesion was consistent with a chordoma. The pathological diagnosis was also reported as a chordoma. 

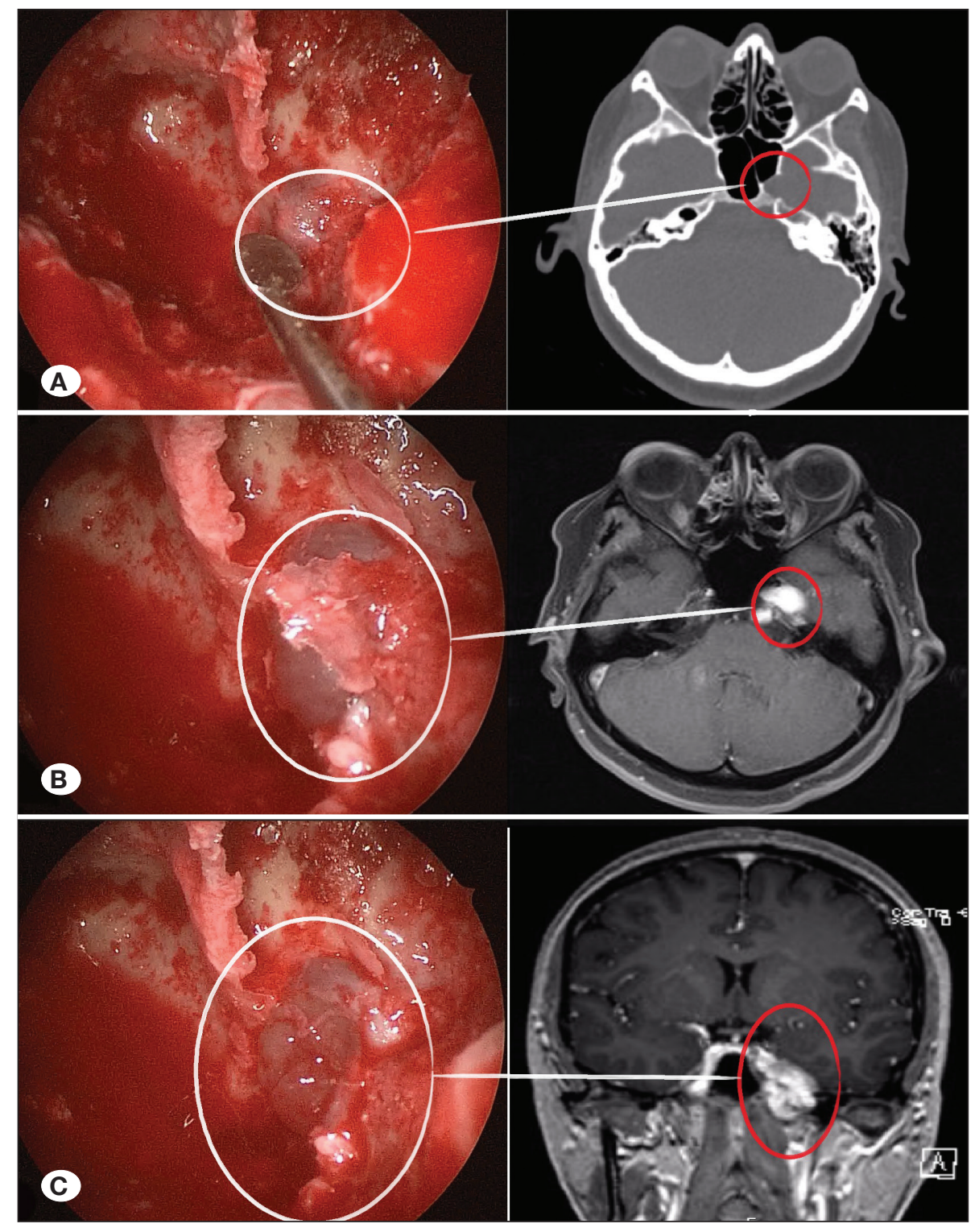

Figure 1: A) Matching of endoscopic image with cranial CT bone window image of Meckel's cave localization lateral to the septum within the sphenoid sinus. B) Matching of tumor tissue access with contrast axial cranial MRI image following removal of the window-shaped bone in the sphenoid sinus.

C) Matching of endoscopic image of tumor tissue with coronal plane of contrast axial cranial MRI image.

\section{DISCUSSION}

In 1984, Kapila et al. reported Meckel's cave as a dural sheath on the gasser ganglion, located on the posterior inferior surface of the cavernous sinus, and on the medial posterior of the medial fossa (7). Primer tumors of Meckel's cave make up less than $0.5 \%$ of all intracranial tumors (11). The majority of Meckel's cave tumors are trigeminal schwannomas (33\%). The other pathologies include meningiomas, invasive pituitary macroadenomas, metastases, epidermoid cysts, lipoma, lymphoma, and other skull base tumors (12). The incidence of trigeminal schwannomas among all intracranial tumors is 0.07 to $0.28 \%$; however, the incidence of all intracranial schwannomas is reported to be between 0.8 to $8 \%$. Trigeminal schwannomas enlarge, spreading from the middle fossa to the posterior fossa and present as a characteristic dumbbell shape on radiological images. However, this may not be the case with smaller lesions as in this case. On MRI, trigeminal schwannomas on T1-weighted imaging appear as hyperintense images, whereas they appear as hyperintense and heterogeneous contrast enhancements on T2- and contrast-enhanced images.

The location of chordomas at the skull base is generally in the neighborhood of the midline of the clivus. In the literature, chordomas are reported to be rarely observed in places such as the intrasellar, suprasellar, tentorium cerebelli, foramen magnum, cervical and thoracic region. Chordomas of the Meckel's cave are rarely observed in the literature. Their radiological characteristics are similar to those of schwannomas, and may be confused with trigeminal schwannoma especially in this region. Although similar cases have been reported in the literature, they are very rare $(4,9)$. The classical means of accessing tumors of Meckel's cave is by the microscopic subtemporal extradural approach. 


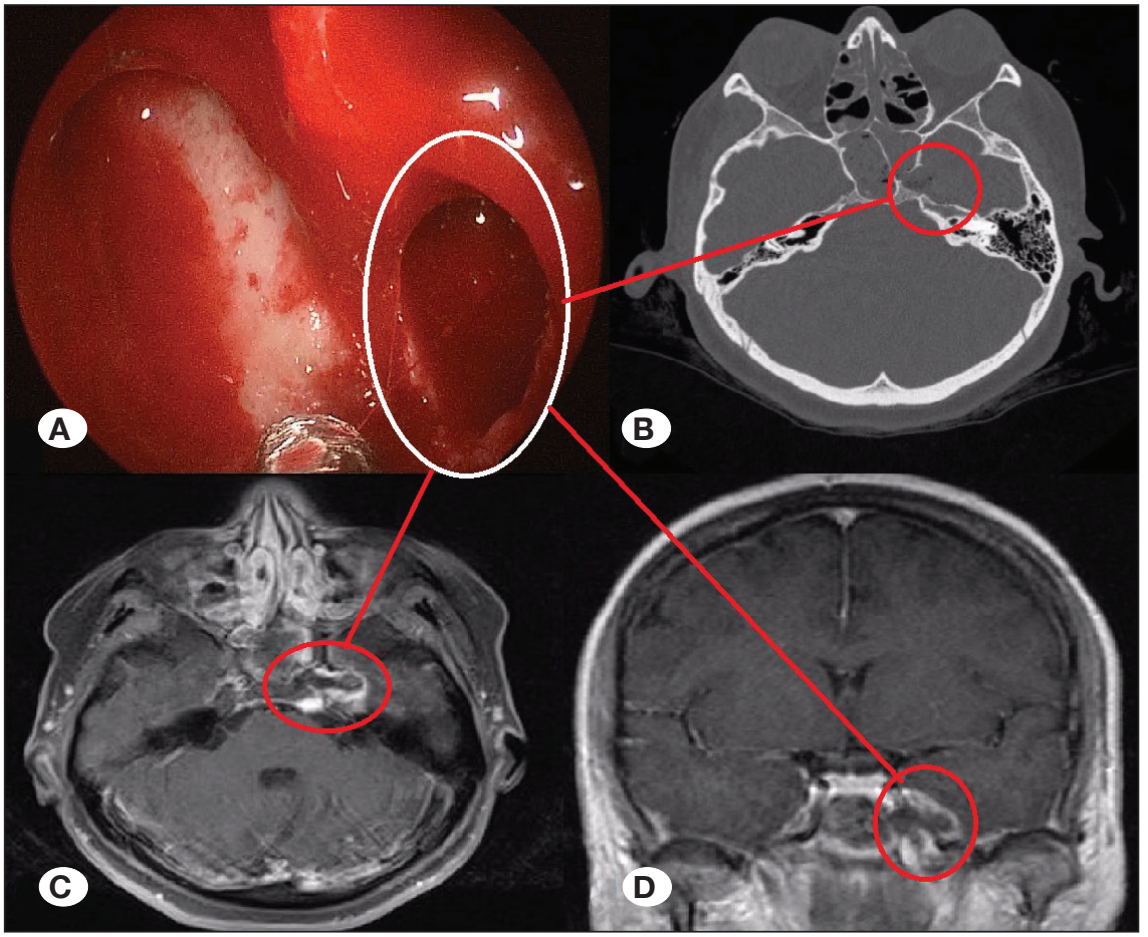

Figure 2: A) Matching of postoperative radiological examination techniques with endoscopic imaging following excision of tumor tissue; B) postoperative CT bone window; C) postoperative contrast cranial MRI image of the axial plane;

D) postoperative contrast cranial MRI image of the coronal plane.

\section{CONCLUSION}

With recent developments in endoscopic technology, there is increasingly frequent use of endoscopic endonasal skull base interventions. In particular, nowadays, endoscopic endonasal skull base surgery has become the gold standard method for accessing sellar, clival, and parasellar masses. As it is particular in this case, we suggest that the use of navigation also continuously improves the reliability of endoscopic surgery by eliminating the misleading effect of anatomical variations.

\section{REFERENCES}

1. Bhat DI, Yasha M, Rojin A, Sampath S, Shankar SK: Intradural clival chordoma: A rare pathological entity. J Neurooncol 96: 287-290, 2010

2. Dahlin DC, MacCharty CS: Chordoma: A study of 59 cases. Cancer 5: 1170-1178, 1952

3. Dow GR, Robson DK, Jaspan T, Punt JA: Intradural cerebellar chordoma in a child: A case report and review of the literature. Childs Nerv Syst 19:188-191, 2003

4. Falconi M, Taibah A, Caruso A: Clival chordoma mimicking a trigeminal schwannoma. Otol Neurotol 22:706-707, 2001

5. Inci S, Palaoglu S, Onol B, Erbengi A: Low cervical chordoma: Case report. Spinal Cord 34:358-360, 1996

6. Ito E, Saito K, Nagatani T, Ishiyama J, Terada K, Yoshida M, Wakabayashi T: Intradural cranial chordoma. World Neurosurg 73:194-197, 2010

7. Kapila A, Chakeres DW, Blanco E: The Meckel cave: Computed tomographic study. Part I: Normal anatomy; Part II: Pathology. Radiology 152:425-433, 1984
8. Kondziolka D, Lunsford LD, Flickinger JC: The role of radiosurgery in the management of chordoma and chondrosarcoma of the cranial base. Neurosurgery 29: 38-45; discussion 45-46, 1991

9. Lu CY, Chai CY, Chiang IC: Chordoma mimicking the trigeminal schwannoma: A case report. Clin Imaging 28:187-190, 2004

10. Nishigaya $\mathrm{K}$, Kaneko $\mathrm{M}$, Ohashi $\mathrm{Y}$, Nukui $\mathrm{H}$ : Intradural retroclival chordoma without bone involvement: No tumor regrowth 5 years after operation. Case report. J Neurosurg 88: 764-768, 1998

11. Obeng K, Rumboldt Z, Tuite G, Welsh CT, Patel S: Atypical cystic meningioma of the trigeminal nerve in a pediatric patient. AJNR 29: 398-399, 2008

12. Ostehus DR, Van Loveren HR, Friedman RA: Trigeminal schwannoma. Am J Otol 20:551-552, 1999

13. Roberti F, Sekhar LN, Jones RV, Wright DC: Intradural cranial chordoma: A rare presentation of an uncommon tumor. Surgical experience and review of the literature. J Neurosurg 106: 270-274, 2007

14. Samii M, Cheatham ML, Becker DP: Chordomas, chondroma and chondrosarcomas. In: Atlas of Cranial Base Surgery. UCLA and Hannover Experience. Philadelphia: WB Saunders, 1995: 186-187

15. Wolfe JT, Scheithauer BW: Intradural chordoma or giant ecchordosis physaliphora? Report of two cases. Clin Neuropathol 6: 98-103, 1987 